\title{
DISCUSIONES SOBRE AUTONOMÍA EN LA RECEPCIÓN DE LOS APORTES DEPENDENTISTAS AL CAMPO DE LAS RELACIONES INTERNACIONALES $Y$ CONTRIBUCIONES RECIENTES DESDE EL ENFOQUE DE CADENAS GLOBALES DE VALOR
}

\section{DISCUSSIONS ON ANTONOMY IN THE RECEPTION IF THE DEPENDENCY CONTRIBUTIONS TO THE FIELD OF INTERNATIONAL RELATIONS AND RECENT CONTRIBUtions fROM the GLobal VALUE Chains APPROCH}

Mariano Treacy

\begin{abstract}
RESUMEN
La teoría de la dependencia es reconocida en el mundo de las Relaciones Internacionales como una de las principales contribuidoras al Campo desde los países de América Latina. En este trabajo nos proponemos el objetivo de identificar las características de la recepción de los aportes dependentistas bajo la hipótesis de que esta ha estado mediada por los desarrollos de la Escuela de la Autonomía Latinoamericana y no por las contribuciones de quienes son considerados los autores dependentistas canónicos en el campo de la Economía Política. Lo que observaremos es que el concepto de autonomía que se trabaja desde la escuela latinoamericana es considerablemente diferente del que se trabaja desde la Economía Política. Además de un balance crítico, se realiza una propuesta para incorporar al marco del análisis de la autonomía la problemática de las cadenas globales de valor.
\end{abstract}

\section{PALABRAS CLAVE}

Dependencia, Autonomía, Relaciones Internacionales, Cadenas Globales de Valor

\section{ABSTRACT}

The dependency theory is recognized in the world of International Relations as one of the main contributors to the field from Latin American countries. In this paper we propose the objective of identifying the characteristics of the reception of dependentist contributions under the hypothesis that this has been mediated by the developments of the Latin American school of Autonomy and not by the contributions of those who are considered the canonical dependentist authors in the field of Political Economy. What we will observe is that the concept of autonomy used by the Latin American school is considerably different from the one used by the Political Economy. In addition to a critical balance, a proposal is made to incorporate the problem of global value chains into the autonomy analysis framework.

\section{KEY WORDS}

Dependency, Autonomy, International Relations, Global Value Chains

\footnotetext{
"Una versión preliminar de este trabajo, que forma parte del trabajo de tesis doctoral del autor, fue presentada en el Encuentro sobre actualidad y vigencia de la teoría marxista de la dependencia en la Universidad Nacional de La Plata en julio de 2017. El autor agradece la paciente lectura y los comentarios de Ariel Slipak a una versión previa de este trabajo y lo exime de las opiniones vertidas y de los errores que pudieran encontrarse aquí.
} 
Revista Aportes para la Integración Latinoamericana Año XXIV, Nº 38/Junio 2018, ISSN 2468-9912. DNDA: 5355295 en línea

Mariano Treacy

Discusiones sobre la autonomía en la recepción de los aportes dependentistas al campo de las Relaciones Internacionales y contribuciones recientes desde el enfoque de Cadenas Globales de Valor

Págs. 45-67

\section{INTRODUCCIÓN}

Si bien el grueso del conocimiento que tiene el mundo sobre la periferia es producido por académicos de los países centrales ${ }^{1}$ que construyen cierta hegemonía con categorías derivadas de su propia cosmovisión (Tickner, 2009), la Teoría de la Dependencia ha realizado aportes sustanciales que hasta el día de hoy tienen relevancia a la hora de problematizar la Economía Política Internacional (Tussie, 2015). En la actualidad, la Teoría de la Dependencia es reconocida en el mundo de las Relaciones Internacionales como una de las principales contribuyentes al campo ${ }^{2}$ desde los países de América Latina (Tickner, 2009, 2011; Tussie 2015; Briceño Ruiz, y Simonoff, 2017).

Los aportes de la Teoría de la Dependencia a la caracterización de la geografía, la geopolítica y la economía política de las Relaciones Internacionales han contribuido a la incorporación de una cosmovisión propia de los países subdesarrollados (Tickner, 2013). Lejos de representar una contribución marginal, el ejercicio de "pensar las Relaciones Internacionales" desde la periferia favoreció que parte de la academia y de la diplomacia internacional absorbiera algunos tópicos que difícilmente hubiesen adquirido esa relevancia de no haber sido enunciados desde el sur global. Entre estos tópicos, se destacan las relaciones de poder existentes en los distintos niveles del sistema y las estructuras sociales, económicas y políticas que las reproducen (Tussie, 2015).

La recepción ${ }^{3}$ de las contribuciones dependentistas en el campo de las relaciones internacionales ha estado mediada por los aportes de la Escuela de la Autonomía

\footnotetext{
${ }^{1}$ Dominadas tradicionalmente por enfoques provenientes de países ricos o desarrollados, las teorías de las relaciones internacionales más difundidas delimitan la agenda de discusión y establecen las bases conceptuales desde donde se piensa la política exterior con un sesgo derivada del carácter "céntrico" de su lugar de enunciación (Tickner, 2011).

${ }^{2}$ En este trabajo se utiliza el concepto de Campo siguiendo a Pierre Bourdieu (2002). La producción científica en disciplinas como la Economía o subdisciplinas como la Economía, Política Internacional, las Relaciones Internacionales o la Integración Regional constituye un campo intelectual con sus propias especificidades (reglas académicas de acceso, reglas y patrones de ascenso a las posiciones de poder dentro de él, etc.). Este concepto permite vincular la creación simbólica de los individuos con el contexto de la producción, los debates políticos y las tensiones sociales en que se encuentran inmersos. El campo es por lo tanto una arena de disputa, un espacio de lucha donde se determina la producción de bienes simbólicos que se transformará en hegemónica, con consecuencias sobre la apropiación en la sociedad civil, en las clases dominantes y en los sectores subordinados (Barneix, Forcinito y Treacy, 2014).

3 "El concepto de recepción remite a un proceso mayor de producción / difusión intelectual en el que es necesario discriminar (analíticamente) a productores, difusores, receptores y consumidores de las ideas. Dentro del proceso global de producción y circulación de las ideas podemos distinguir no etapas temporales sucesivas sino distintos momentos, a cada uno de los cuales corresponde cierto tipo de intelectuales" (Tarcus, 2007: 30).
} 

Año XXIV, N 38/Junio 2018, ISSN 2468-9912. DNDA: 5355295 en línea

Mariano Treacy

Discusiones sobre la autonomía en la recepción de los aportes dependentistas al campo de las Relaciones Internacionales y contribuciones recientes desde el enfoque de Cadenas Globales de Valor

Págs. 45-67

Latinoamericana, cuyos principales exponentes fueron Carlos Puig y Helio Jaguaribe, que posicionaron sus contribuciones en los campos de la Ciencia Política, las Relaciones Internacionales y la Política Exterior. Desde las Relaciones Internacionales, se suelen tomar sus contribuciones como un punto de partida para evaluar el aporte de la Teoría de la Dependencia al campo. Como veremos, esta apropiación tiene la ventaja de que ha permitido problematizar la autonomía del estado, el rol de las élites y los condicionantes de la política exterior incorporando una perspectiva latinoamericana en un campo liderado por contribuciones del norte global.

Sin embargo, la reducción que se hace en esta recepción desde las Relaciones Internacionales al identificar la Teoría de la Dependencia casi exclusivamente con la escuela autonomista latinoamericana ha tenido consecuencias negativas que en este trabajo se intentan identificar. Básicamente, lo que se plantea en esta investigación es que se han dejado de lado elementos centrales vinculados a la economía política internacional dependentista, donde los aportes de Ruy Mauro Marini (1973, 2008), Theotonio Dos Santos (1971, 2011), Agustín Cueva (1979) y Vania Bambirra (1999) han sido centrales.

El objetivo de este trabajo consiste, por lo tanto, en realizar una contribución crítica de la recepción de la Teoría de la Dependencia por parte del campo de las Relaciones Internacionales, argumentando que la identificación de la dependencia con la escuela autonomista latinoamericana ha dejado de lado las condiciones estructurales que se enfatizan desde la Economía Política. Con este objetivo, se reconstruyen tanto las contribuciones de la escuela de la autonomía latinoamericana como de la teoría marxista de la dependencia y se identifican los elementos recogidos y relegados a un segundo plano en la recepción y apropiación. Finalmente se realiza un balance "crítico" de esta apropiación y se ofrece una propuesta de incorporar al marco del análisis de la autonomía la problemática de las cadenas globales de valor, que podría representar un aporte al campo en el marco de la configuración de la acumulación del capital a escala global actual. 

Año XXIV, Nº 38/Junio 2018, ISSN 2468-9912. DNDA: 5355295 en línea

Mariano Treacy

Discusiones sobre la autonomía en la recepción de los aportes dependentistas al campo de las Relaciones Internacionales y contribuciones recientes desde el enfoque de Cadenas Globales de Valor

Págs. 45-67

\section{Los APORTES DE LA teoría de LA DEPENDENCIA AL CAMPO de LAS RELACIONES INTERNACIONALES}

Tanto el Estructuralismo de la Comisión Económica para América Latina y el Caribe (CEPAL) como la Teoría de la Dependencia, estudiaron desde la Economía Política las características y obstáculos estructurales del desarrollo económico en la región. El Estructuralismo cepalino ${ }^{4}$ enfatizó el deterioro de los términos del intercambio (Prebisch), la heterogeneidad estructural (Pinto) y la desigualdad en la distribución del ingreso (Furtado) como algunos de los factores que configuraban la relación centro/periferia que reproducía el patrón desarrollo/subdesarrollo. La comprensión del fenómeno del subdesarrollo en el contexto histórico en el que Prebisch y la CEPAL formularon sus primeras hipótesis condujo a la postulación de que la única estrategia posible para que un país periférico pudiera salir del atraso era la industrialización. El modelo desarrollista de industrialización por sustitución de importaciones (ISI), vigente en América Latina hasta entrada la década del setenta, expresó la necesidad de conquistar mayores niveles de autonomía con respecto a las necesidades de importaciones de bienes y de tecnología del exterior (Tickner, 2009). Como se puede observar, en el diagnóstico Estructuralista de los problemas del desarrollo en América Latina el concepto de autonomía aparece ligado a la necesidad económica de romper la dependencia de las importaciones de bienes de capital y tecnología. La construcción de un proyecto autónomo, por lo tanto, se basaba en la sustitución de esas importaciones y la consolidación del mercado local.

Las Teorías de la Dependencia nacen en los años sesenta con el objetivo de identificar las razones que obstaculizaban el desarrollo económico en América Latina una vez avanzado y consolidado el proceso de industrialización por sustitución de importaciones y profundizada la penetración del capital extranjero en las estructuras productivas locales. En este contexto, estas perspectivas fueron construidas como respuesta a la incapacidad del Estructuralismo cepalino y de las teorías desarrollistas en general, de dar cuenta de las crisis económicas cíclicas por la que la mayoría de los países latinoamericanos

\footnotetext{
${ }^{4}$ El Estructuralismo cepalino o Estructuralismo Latinoamericano es una corriente heterodoxa dentro del campo de la Economía Política que intenta pensar desde la región las causas del atraso económico proponiendo recomendaciones de políticas públicas. Se consolida luego de la creación de la Comisión Económica para América Latina y el Caribe (CEPAL) en Santiago de Chile en 1948. Entre sus principales referentes se cuentan Raúl Prebisch, Celso Furtado, Aníbal Pinto y Osvaldo Sunkel, entre otros.
} 

Año XXIV, Nº 38/Junio 2018, ISSN 2468-9912. DNDA: 5355295 en línea

Mariano Treacy

Discusiones sobre la autonomía en la recepción de los aportes dependentistas al campo de las Relaciones Internacionales y contribuciones recientes desde el enfoque de Cadenas Globales de Valor

Págs. 45-67

atravesaron desde comienzos de la década del 60 y de los límites que manifestaron para lograr un crecimiento sostenido con una distribución del ingreso cada vez más progresiva.

La Teoría Marxista de la Dependencia (Marini, Dos Santos, Bambirra), en particular, fue aquella que, a diferencia de los enfoques weberianos de la dependencia (Cardoso y Faletto) o desarrollistas (Sunkel, Pinto), planteó la imposibilidad de desarrollo económico de los países periféricos en el marco del sistema capitalista (Palma, 1987). El clima de época estaba marcado por el momento de mayor expansión del campo socialista en plena guerra fría y por la irrupción de la Revolución Cubana en América Latina y de procesos de descolonización en África y Asia (Borón, 2008).

La Teoría de la Dependencia tomó en cuenta los desarrollos cepalinos y les confirió un carácter sistémico y relacional, en el sentido de que las posibilidades de desarrollo y de espacios de autonomía en un espacio local o regional se encuentran vinculadas de forma sistémica al modo de producción, circulación y consumo en un nivel global. En cierto sentido, se hace énfasis en el factor externo, en las relaciones de poder que se establecen en el mercado mundial y en factores estructurales que condicionan las particularidades nacionales y las posibilidades que tiene una nación particular de salir del atraso.

La autonomía, para la Teoría de la Dependencia, no va a ser ya una opción de modelo de desarrollo de industrialización por sustitución de importaciones, sino una relación de poder en donde la estructura jerárquica del sistema internacional va a cumplir una función principal. Existen actores con más peso, como Estados con funciones hegemónicas (Estados Unidos, la URSS) o potencias mundiales entre las que se produce una Cooperación antagónica ${ }^{5}$ o actores no estatales como Empresas Transnacionales, que van construyendo e imponiendo las reglas en la que se desarrolla la acumulación del

\footnotetext{
${ }^{5}$ El concepto de Cooperación antagónica, elaborado por Marini es utilizado para explicar las relaciones entre las potencias mundiales explicadas por intereses y pretensiones particulares. Estas relaciones cubren el abanico completo de integración-cooperación-competencia-conflicto (Slipak, 2016). Marini lo utiliza para ilustrar las relaciones que existen, por ejemplo, entre Estados Unidos y Francia: "los demás países industrializados, (...) sometidos a la penetración de las inversiones norteamericanas, se volvieron a su vez centros de exportación de capitales y extendieron simultáneamente sus fronteras económicas, dentro del proceso ecuménico de la integración imperialista. Las tensiones que intervinieron entre esos varios centros integradores, de desigual grandeza (como, por ejemplo, Francia y Estados Unidos), aunque no puedan, como en el pasado, llegar a la hostilidad abierta y tengan que mantenerse en el marco de la cooperación antagónica" (Marini, 2008).
} 

Año XXIV, Nº 38/Junio 2018, ISSN 2468-9912. DNDA: 5355295 en línea

Mariano Treacy

Discusiones sobre la autonomía en la recepción de los aportes dependentistas al campo de las Relaciones Internacionales y contribuciones recientes desde el enfoque de Cadenas Globales de Valor

Págs. 45-67

capital en el plano global. Estas reglas se van cristalizando, con el correr de los años, en la construcción de normativas que se van imponiendo en organismos internacionales y mutilaterales $^{6}$. La autonomía es por lo tanto la contracara de la dependencia, ya que expresa la imposibilidad de pensarla como una estrategia soberana de política exterior.

Con el avance del neoliberalismo y la consolidación de la mundialización del capital desde los años 80 , las ideas y Teorías de la Dependencia y de la autonomía fueron entrando en desuso, lo que no implica que no puedan ser reformuladas para entender las relaciones de poder que existen en el mercado mundial en la actualidad

\section{Creación e institucionalización del Consejo de Salud SuRamercano y sus ÓRGANOS SUBSIDIARIOS (GT, RES E ISAGS)}

\section{La construcción de la Autonomía desde el Sur Global}

Algunos referentes del campo de las Relaciones Internacionales (Tickner, 2009, 2011, 2013; Tussie, 2015; Briceño Ruiz y Simonoff, 2017; Miguez, 2017) plantean que la región contribuyó mediante el desarrollo de la escuela o doctrina de la autonomía, que tomó aportes del pensamiento de la CEPAL y de la Teoría de la Dependencia (Economía Política), así como también contribuciones del realismo y de la interdependencia (Relaciones Internacionales).

Así como el estructuralismo de la CEPAL y la Teoría de la Dependencia realizaron una elaboración de una teoría del subdesarrollo desde la región contrapuesta en muchos aspectos a la economía del desarrollo liberal (Rostow) o keynesiana (Hirschman, Lewis, Nurkse, Myrdal), en el campo de las Relaciones Internacionales se les reconoce haber jerarquizado el problema de la autonomía en contraste con las dominantes perspectivas Liberales o Realistas, cuyo énfasis había sido puesto en la interdependencia (Keohane y Nye), la hegemonía (Cox, Bieler y Morton) o el interés nacional y el equilibrio de poder interestatal (Morgenthau, Waltz).

\footnotetext{
${ }^{6}$ Con distintas funciones entre sí, organismos como el Fondo Monetario Internacional (FMI), el Banco Mundial (BM), la Organización de las Naciones Unidas (ONU), el Acuerdo General Sobre Aranceles y Comercio (GATT), la Organización Mundial del Comercio (OMC), los Tratados de Libre Comercio (TLC) o Tratados Bilaterales de Inversión (TBI), los acuerdos de integración regional o grupos como el G-20 son todos espacios en donde los Estados ceden parte de su soberanía con el objetivo de participar de un consorcio global del que esperan sacar algún tipo de beneficio (Treacy, 2016).
} 
Revista Aportes para la Integración Latinoamericana Año XXIV, Nº 38/Junio 2018, ISSN 2468-9912. DNDA: 5355295 en línea

Mariano Treacy

Discusiones sobre la autonomía en la recepción de los aportes dependentistas al campo de las Relaciones Internacionales y contribuciones recientes desde el enfoque de Cadenas Globales de Valor

Págs. 45-67

A diferencia de la "Soberanía"7 (tópico largamente abordado en la academia en Estados Unidos y Europa), la cuestión de la Autonomía había sido históricamente una preocupación específicamente latinoamericana, ya que "ofrece una óptica distinta para pensar sobre política exterior y política internacional" (Tickner, 2014) rechazando la noción de soberanía que asume que todos los Estados soberanos, por el hecho de ser independientes, poseen autonomía (Briceño Ruiz, J. y Simonoff, A., 2017).

La autonomía será por lo tanto el principal tópico que se abordará en el marco de las contribuciones de la Teoría de la Dependencia al campo de las Relaciones Internacionales. José Briceño Ruiz y Alejandro Simonoff (2017), Arlene Tickner (2009, 2011, 2013), Diana Tussie (2015) y María Cecilia Miguez (2017) reconstruyen los aportes de la Teoría de la Dependencia a este campo considerando principalmente (aunque no de forma exclusiva) las contribuciones de la escuela de la autonomía, cuyos principales exponentes fueron Juan Carlos Puig (Argentino, 1928-1989) y Helio Jaguaribe (Brasilero, 1926). El objetivo que persiguen los autores es demostrar que el autonomismo latinoamericano realizó un aporte original desde el Sur Global a las teorías de las relaciones internacionales, tomando cuestiones del realismo (Morgenthau, Waltz) y el liberalismo (Moravcsik, Nye Jr., Keohane) pero también reinterpretando críticamente las contribuciones latinoamericanas de la CEPAL y de la(s) Teoría(s) de la Dependencia ${ }^{8}$.

El problema de la autonomía será por lo tanto el aporte del Sur Global a las Relaciones Internacionales dominado por enfoques realistas que ponen el eje en la hegemonía y el

\footnotetext{
${ }^{7}$ Según el Derecho Internacional, la soberanía tiene carácter constitucional y funcional e implica la igualdad de los Estados, la independencia y el deber de no intervención. La Resolución 2625 (XXV) de la Asamblea General de la Organización de las Naciones Unidas (ONU) en 1970 estableció que "Todos los Estados gozan de la igualdad soberana. Tienen iguales derechos e iguales deberes y son por igual miembros de la comunidad internacional, pese a las diferencias de orden económico, social, político o de otra índole. En particular, la igualdad soberana comprende los elementos siguientes: i) Los Estados son iguales jurídicamente; ii) Cada Estado goza de los derechos inherentes a la plena soberanía; iii) Cada Estado tiene el deber de respetar la personalidad de los demás Estados; iv) la integridad territorial y la independencia política del Estado son inviolables; v) Cada Estado tiene el derecho a elegir y a llevar adelante libremente su sistema político, social, económico y cultural; vi) Cada Estado tiene el deber de cumplir plenamente y de buena fe sus obligaciones internaciones y de vivir en paz con los demás Estados".

${ }^{8}$ La recepción que estos autores realizan del Estructuralismo y de la teoría de la dependencia se produce "mediada" por la lectura que realizan Puig y Jaguaribe. Como mencionamos previamente, en el campo de la economía política, estos autores no forman parte del cuerpo canónico de autores ni estructuralistas ni dependentistas. Esta lectura "mediada" por las interpretaciones de la dependencia y el estructuralismo por parte de la Escuela de la Autonomía se puede corroborar observando la sobreabundancia de citas de estos autores en la bibliografía, y la ausencia casi absoluta de citas de autores estructuralistas "clásicos" como Prebisch, Furtado, Sunkel, Ferrer o Pinto o dependentistas como Cardoso y Faletto, Marini, Dos Santos, Bambirra, Cueva o Gunder Frank.
} 

Año XXIV, Nº 38/Junio 2018, ISSN 2468-9912. DNDA: 5355295 en línea

Mariano Treacy

Discusiones sobre la autonomía en la recepción de los aportes dependentistas al campo de las Relaciones Internacionales y contribuciones recientes desde el enfoque de Cadenas Globales de Valor

Págs. 45-67

equilibrio de poderes del sistema interestatal o enfoques liberales que enfatizan la interdependencia, la competencia y la cooperación entre actores estatales y no estatales. El orden internacional no está cristalizado (ya que puede haber movimientos) pero sí jerarquizado (ya que existen asimetrías sistémicas). En el orden interestatal hay reglas escritas y no escritas. Si bien los Estados más poderosos tienen un papel preponderante en el establecimiento de esas normas, no todos tienen los mismos derechos en el sistema interestatal ya que unos pocos tienen el poder de forjar las reglas de juego mientras que la mayoría debe comportarse según las normas establecidas (Escudé, 2012).

La relación entre autonomía y poder va a ser evidente para este enfoque, ya que su naturaleza (es decir, de dónde proviene) y los lazos que genera configuran el escenario internacional, lo que influye en el margen de maniobra ${ }^{9}$ del resto de los actores no dominantes (estatales y no estatales) sobre su capacidad de actuar en función de sus propios intereses. Sin embargo, esta relación no será ni lineal ni absoluta como se plantea desde la corriente realista, donde los Estados son los actores más importantes del sistema internacional y sus acciones se entienden solo en el marco de la defensa del interés nacional, la búsqueda de seguridad y de poder y el equilibrio de poderes (Legler, 2016).

La Escuela Latinoamericana de la Autonomía construye un "modelo híbrido" (Tickner, 2011) para postular un sistema global en el que existen interacciones donde los actores no estatales tienen tanta relevancia como los estatales y donde se establecen relaciones jerárquicas entre las naciones derivadas de su relativo poder económico y militar (Miguez, 2017). El peso de los actores transnacionales, intergubernamentales y transgubernamentales se deriva de la "creciente transnacionalización del mundo" y del control que ejercen sobre la innovación tecnológica. Aquí, el Estado no es considerado un actor único ni racional sino que los grupos de presión o élites se disputan su control y delinean las diversas formas de inserción ${ }^{10}$. Es decir, que el "interés nacional" se define

\footnotetext{
${ }^{9}$ Carlos Escudé suele utilizar el concepto margen de maniobra o de negociación como sinónimo de autonomía. Si bien reconoce distintos grados, el autor es crítico de quienes lo sobreestiman o el margen de maniobra de los Estados Débiles y de quienes no distinguen entre la autonomía que un Estado posee y el uso que le da a la misma (Escudé, 2012).

10 Para Puig (1984) existen cuatro tipos "ideales" de inserción en función del rol que le asignen las élites a su estado en el sistema internacional. Si no poseen proyecto propio y se subordinan al poder de la gran potencia, la inserción es de un tipo de "dependencia paracolonial". Si poseen un proyecto propio, aunque sea
} 

Año XXIV, Nº 38/Junio 2018, ISSN 2468-9912. DNDA: 5355295 en línea

Mariano Treacy

Discusiones sobre la autonomía en la recepción de los aportes dependentistas al campo de las Relaciones Internacionales y contribuciones recientes desde el enfoque de Cadenas Globales de Valor

Págs. 45-67

como un resultado de "la base material o ideacional de las élites para conservar sus beneficios y poderes en el Estado-nación" (Briceño Ruiz, J. y Simonoff, A., 2017).

El sistema internacional se caracteriza por lo tanto por condiciones asimétricas y una tensión de poder derivada de la existencia de actores con distintas jerarquías, donde la lógica de conflicto no conduce necesariamente al equilibrio. En este marco, la "dependencia ${ }^{11}$ expresa, para Jaguaribe, una situación de países que poseen soberanía estatal, órganos propios de gobierno y voz como interlocutores de otros Estados y organismos intemacionales pero que no disponen de requisitos para ostentar ni primacía regional ni general. Como podemos observar, la definición de dependencia y de autonomía que presentan Puig y Jaguaribe, si bien tiene algún punto de vinculación con la trabajada desde la Economía Política, se vincula mucho más a las discusiones del campo de la Ciencia Política, las Relaciones Internacionales y la Política Exterior.

Los países periféricos no pueden abstraerse de las condiciones que impone el sistema internacional y deben formular estrategias activas. Para Jaguaribe, la autonomía, en este contexto, será un producto de la "viabilidad nacional", que expresará tanto cuestiones estructurales (como la existencia de recursos económicos, humanos, desarrollo tecnológico y cohesión sociocultural) como políticas (de alianzas de cooperación con las potencias centrales, secundarias o subordinadas). Para Puig, además de la viabilidad nacional y una cantidad suficiente de recursos domésticos, la autonomía requiere un compromiso explícito por parte de las élites de que la autonomía tiene un valor intrínseco (Tickner, 2011).

Para la Escuela Latinoamericana, por lo tanto, la autonomía va a constituir una estrategia específica de política exterior para preservar cierto supuesto "interés nacional" en el sistema internacional, representando un factor indispensable para el desarrollo y para reducir la vulnerabilidad derivada de la dependencia ${ }^{12}$.

dependiente, la inserción es de tipo "dependencia racionalizada". Si tienen un proyecto autonomista la inserción es de tipo "autonomista heterodoxo". Por último, si plantean un proyecto que lleve a la ruptura estratégica con las potencias centrales la inserción es de tipo "autonomista secesionista".

${ }^{11}$ El uso de comillas responde a que en este apartado me estoy refiriendo al modo en que fue apropiado ese término por la Escuela de la Autonomía y por el campo de las relaciones internacionales.

12 "La autonomía no sólo se veía como factor indispensable para el desarrollo sino que el concepto también empezó a vincularse con la política exterior. A nivel nacional, la autonomía se consideraba como una 

Año XXIV, Nº 38/Junio 2018, ISSN 2468-9912. DNDA: 5355295 en línea

Mariano Treacy

Discusiones sobre la autonomía en la recepción de los aportes dependentistas al campo de las Relaciones Internacionales y contribuciones recientes desde el enfoque de Cadenas Globales de Valor

Págs. 45-67

La estrategia autonomista se centra en la idea de garantizar la independencia del Estado para asegurar formas de desarrollo no dependiente, generando instrumentos para defender la soberanía, controlar actividades de empresas extranjeras, afianzar capacidades locales y defender intereses nacionales frente a las potencias mundiales (Tickner, 2011). Para lograr ensanchar el margen de autonomía en los países de la periferia, una estrategia autonomista debe considerar el papel de las élites como un factor clave (Briceño Ruiz, J. y Simonoff, A., 2017). La autonomía pasa a concebirse como estrategia de política exterior y no como una consecuencia de una condición estructural de dependencia.

\section{III. ¿FALTA DE AUTONOMÍA COMO UNA CONDICIÓN ESTRUCTURAL DE DEPENDENCIA O COMO UNA ESTRATEGIA POSIBLE DE POLÍTICA EXTERIOR?}

La Escuela Latinoamericana de la Autonomía, con los aportes de Puig y Jaguaribe, había derivado en la necesidad de proyectos autonómicos de las asimetrías que caracterizan el sistema internacional, tomando en cuenta los aportes del Estructuralismo de la CEPAL y de las teorías de la dependencia. El desarrollo de la periferia debía darse mediante una estrategia de autonomización, modificando la situación de dependencia, removiendo los condicionantes internos y promoviendo el acceso de las grandes mayorías al dominio del Estado. Esta estrategia se basaría en la conquista de una "autonomía periférica" optimizando los principales intereses de un país en el ámbito internacional considerando sus condiciones internas como recursos humanos, naturales y tecnológicos (viabilidad nacional) y los medios de acción de que dispone dada su situación geopolítica y sus relaciones internacionales con la(s) superpotencia(s) (permisividad internacional), promoviendo una inserción que permita incrementar la autonomía, participando en la construcción de los regímenes internacionales (gobernanza global) ${ }^{13}$ (Briceño Ruiz y Simonoff, 2017).

salvaguardia contra los efectos negativos de la dependencia; a nivel externo, esta se vio como un instrumento para afirmar intereses en el sistema internacional. Para ello se debía separar en cada caso el interés nacional de la potencia dominante" (Tussie, 2015: 163).

${ }^{13}$ A diferencia de la estrategia de "autonomía periférica" que proponen Puig y Jaguaribe, Escudé (2012) planteará la estrategia del "realismo periférico" asociada a establecer alianzas con las principales potencias y obedecerlas. Esto se deriva de su comprensión de las jerarquías de Estados que diferencia aquellos que comandan, aquellos que obedecen y aquellos que no pueden mandar pero se niegan a obedecer. 

Año XXIV, N 38/Junio 2018, ISSN 2468-9912. DNDA: 5355295 en línea

Mariano Treacy

Discusiones sobre la autonomía en la recepción de los aportes dependentistas al campo de las Relaciones Internacionales y contribuciones recientes desde el enfoque de Cadenas Globales de Valor

Págs. 45-67

Como vimos, el Modelo híbrido latinoamericano planteado por la Escuela de la Autonomía, si bien tomaba en cuenta los aportes de la dependencia, construía su idea de "interés nacional" de modo realista, ya que concebía como posibilidad un proyecto autonómico de las elites para conservar sus beneficios y poderes en el Estado.

La dependencia es un concepto que nos permite observar la relación que existe entre la reglamentación internacional en materia comercial y de inversiones, el rol de la empresa transnacional, la división global del trabajo y la repartición de los "beneficios" de la integración funcional neoliberal. Para la Teoría de la Dependencia, la falta de autonomía es consecuencia de una situación estructural de dependencia: de sus élites respecto del capital extranjero; del estado respecto de sus élites; del "proyecto nacional" respecto de la acumulación del capital a escala mundial, etc.

En este sentido, uno de los principales aportes de la Teoría de la Dependencia a la Economía Política Internacional fue una identificación temprana de los efectos no deseados de la inversión extranjera directa sobre la estructura productiva, la estabilidad macroeconómica y las alianzas políticas con las élites locales y los efectos de esto sobre las estrategias de desarrollo. Dos Santos (2011), uno de los padres fundadores de la Teoría de la Dependencia, ya había argumentado que uno de los principales obstáculos al desarrollo era precisamente la subordinación de las élites locales al proyecto de desarrollo del capital extranjero. Esta subordinación no era solo económica sino que obviamente tenía efectos políticos de magnitud, dificultando el establecimiento de un proyecto de "interés nacional".

La política exterior se encuentra condicionada por factores internos y la autonomía del estado está marcada por un margen de acción definido por la complejidad de la estructura productiva que configura un determinado bloque en el poder. Cuanto mayor es la complejidad de la estructura productiva, mayor será la autonomía relativa del estado para establecer su política exterior. Este tipo de autonomía va a estar influida por las características del comercio exterior, la participación del capital extranjero en la acumulación, la disponibilidad de recursos naturales, los grados de endeudamiento, la política de defensa y las alianzas políticas con determinados socios, entre otras variables (Miguez, 2017). 

Año XXIV, Nº 38/Junio 2018, ISSN 2468-9912. DNDA: 5355295 en línea

Mariano Treacy

Discusiones sobre la autonomía en la recepción de los aportes dependentistas al campo de las Relaciones Internacionales y contribuciones recientes desde el enfoque de Cadenas Globales de Valor

Págs. 45-67

El comercio mundial está caracterizado por relaciones de interdependencia entre distintas economías. Estas relaciones se tornan dependientes cuando el ciclo de reproducción del capital de unos tiene la capacidad de "expandirse y autoimpulsarse" mientras que en los otros sólo lo puede hacer como reflejo de esta expansión (Dos Santos, 2011). Las asimetrías se derivan no solo del ejercicio de una posición dominante en función del predominio tecnológico, comercial, financiero y sociopolítico que poseen los estados y capitales de los países centrales, sino de la debilidad de la integración productiva en los países periféricos, que los sitúa en una situación de mayor vulnerabilidad relativa. La configuración específica de este tipo de relaciones de dependencia en esta etapa es un producto histórico de la llegada de inversiones extranjeras a América Latina desde fines de la década del $50^{14}$ y de la forma en que se modificó la acumulación del capital tras la reestructuración productiva desde fines de los 70 .

La industrialización en América Latina permitió homogeneizar los procesos productivos e igualar la productividad y la intensidad del trabajo en algunos nichos, pero no permitió la absorción del conocimiento técnico sino que permitió ubicar el capital sobrante del centro, explotar ventajas específicas y hacer frente a la creciente competencia entre grandes empresas líderes de cada rama (Marini, 2008). La instalación del capital extranjero y el crecimiento industrial que generó, sin embargo, produjo un tipo de articulación dependiente ya que agudizó la heterogeneidad tecnológica, la concentración del capital y los problemas de la balanza de pagos ${ }^{15}$. El desarrollo industrial se concentró en algunos nichos que mantuvieron la dependencia de la importación de bienes de capital, herramientas y tecnología ${ }^{16}$.

En el caso de América Latina, a diferencia de lo ocurrido en el Sudeste Asiático ${ }^{17}$, la instalación de empresas extranjeras no supuso en general la transferencia de la

\footnotetext{
${ }^{14}$ La llegada del capital extranjero en aquel entonces estuvo incentivada por la búsqueda de ventajas de un mercado interno en expansión y para evitar los problemas arancelarios que conllevaba el comercio exterior (Marini, 2008).

${ }^{15}$ Una vez instaladas, estas inversiones generaban necesidades crecientes de importaciones de maquinarias e insumos para crecer, que solo podían satisfacerse con un flujo relativamente estable (y creciente) de divisas. Como el sector exportador no se expandía proporcionalmente a las necesidades de divisas de la industria, se generaban ciclos de stop-n-go marcados por la restricción externa al crecimiento.

${ }^{16}$ Esto se debió principalmente al modo en que la región se insertó en la nueva división global de trabajo, donde los países centrales controlaron el desarrollo tecnológico, concentraron los laboratorios de I+D y seleccionaron qué segmentos productivos se transferirían a la periferia.

${ }^{17}$ Los casos típicos son los de los cuatro "tigres" asiáticos: Corea del Sur, Taiwán, Singapur y Hong Kong.
} 
Revista Aportes para la Integración Latinoamericana Año XXIV, Nº 38/Junio 2018, ISSN 2468-9912. DNDA: 5355295 en línea

Mariano Treacy

Discusiones sobre la autonomía en la recepción de los aportes dependentistas al campo de las Relaciones Internacionales y contribuciones recientes desde el enfoque de Cadenas Globales de Valor

Págs. 45-67

tecnología con la que operaban, ni el desarrollo de encadenamientos y eslabonamientos con proveedores locales, lo que obstruyó el escalamiento productivo (Coatz y Schteingart, 2010). Por lo contrario, el rol del capital extranjero en la industrialización por sustitución de importaciones se limitó muchas veces a la importación por la vía del comercio de insumos y maquinaria y la repatriación de las utilidades generadas, que, acompañadas por el pago de patentes y royalties, y de intereses financieros de la deuda, reproducian las condiciones estructurales de crisis ${ }^{18}$ (Treacy, 2015). Al no modificarse la fuente de las divisas para financiar el proceso manufacturero, la oligarquía diversificada ${ }^{19}$, tradicionalmente exportadora, mantuvo siempre un poder estructural que le permitió sostener sus privilegios, manteniéndose en todos los ciclos políticos dentro del bloque en el poder ${ }^{20}$ (Arceo, 2005).

El modelo de desarrollo, por lo tanto, estuvo basado en exportaciones de productos agrícolas tradicionales y el mantenimiento de una industria dominada por el capital extranjero sin el interés de desarrollar superávit externo ni transferir tecnología. El capital industrial nacional funcionó como un apéndice o como aliado menor en el bloque dominante $^{21}$, lo que le confirió una debilidad política que se mantuvo básicamente en todas las etapas posteriores ${ }^{22}$. Dependiente de las divisas de las exportaciones agrícolas, de los subsidios y los contratos públicos y de las importaciones de maquinaria, insumos y tecnología extranjera, la 'burguesía nacional' no pudo desarrollar un proyecto político independiente de autonomía, ni hegemonizar el bloque en el poder (Cardoso y Faletto, 1969).

\footnotetext{
${ }^{18}$ En la Argentina, la transferencia de excedente en concepto de remisión de utilidades y dividendos, pago de intereses de la deuda externa y fuga de capitales ha totalizado entre 1970 y 2013479.271 millones de USD, lo que equivale a 10.873 millones de USD anuales en promedio, o el 5,8\% del PBI (Treacy, 2015).

${ }_{19}$ Se denomina oligarquía diversificada a aquella fracción empresaria que "por su origen, conformación e intereses se la puede considerar como un sector de la oligarquía local con intereses en la industria, el agro y otras actividades económicas" (Basualdo, 2010: 31).

${ }^{20}$ El peso político del sector agrícola en el bloque en el poder en la Argentina es también una gran diferencia con el caso de los países asiáticos de acelerado desarrollo industrial ya que en todos ellos se dieron reformas agrícolas que liquidaron o disminuyeron considerablemente su importancia y permitieron crear y sostener a una burguesía local con un fuerte interés nacional.

${ }^{21}$ Dos Santos observa frente al golpe militar de 1964 cómo se transforma la burguesía brasileña, que se internacionaliza y se asocia al capital extranjero conformando la nueva "Burguesía Local" asociada, muy distinta de la tradicional oligarquía terrateniente y de la burguesía nacional.

${ }^{22}$ En la Argentina, la excepción sería la salida de la crisis de 2001, cuando el llamado "Grupo Productivo" pudo imponer su programa de salida de la convertibilidad mediante una alianza breve (2003-2007) con sectores populares. El gran capital, si bien se vio desplazado temporalmente, también se vería beneficiado por el nuevo esquema (Constantino y Cantamutto, 2014).
} 

Año XXIV, N 38/Junio 2018, ISSN 2468-9912. DNDA: 5355295 en línea

Mariano Treacy

Discusiones sobre la autonomía en la recepción de los aportes dependentistas al campo de las Relaciones Internacionales y contribuciones recientes desde el enfoque de Cadenas Globales de Valor

Págs. 45-67

Como no pudieron controlar el excedente ni establecer una clara hegemonía política ni desarrollar un sistema nacional de innovación, las elites industriales locales se aliaron a los intereses de la clase capitalista transnacional (Robinson, 2011), buscando sus beneficios por la vía de la especulación financiera, los contratos de obra pública ${ }^{23}$ o las transferencias (vía subsidios, tipo de cambio, tarifas, condonación de deuda).

La dependencia no es por tanto un factor ni exclusivamente externo ni exclusivamente interno, sino que es una condición que configura cierto tipo de estructuras, donde se dificulta acortar la brecha de ingresos y tecnológica, se agudiza el desarrollo geográficamente desigual del capitalismo, se transfiere parte del excedente que se genera internamente y se genera un modelo de desarrollo excluyente, que requiere estratificación del consumo, desigualdad social y precarización laboral para funcionar. Pensar la autonomía y las estrategias autonómicas de política exterior exige entonces, considerar los factores estructurales que la restringen.

\section{DEPENDENCIA Y AUTONOMÍA EN LA ACTUALIDAD: MUNDIALIZACIÓN Y CADENAS DE VALOR}

La globalización se define como una superación progresiva de las fronteras nacionales en el marco del mercado mundial vinculada a una internacionalización creciente de las estructuras de producción, circulación y consumo de bienes y servicios, que altera la geografía política, las Relaciones Internacionales, la organización social, las escalas de valores y las configuraciones ideológicas de cada país (Marini, 2008: 248). La modificación del régimen de acumulación a escala global, que agudizó la tendencia a la mundialización desde fines de los 70 , nos permite rediscutir y reinterpretar las categorías de autonomía y dependencia.

Desde los años 80 se reconfiguran las relaciones de interdependencia debido a una mayor intensidad de las relaciones comerciales, financieras y productivas entre los distintos espacios nacionales. La segmentación internacional, como indica Minian (2009), se orienta hacia la obtención de menores costos de producción (posibilitados en los países emergentes) y economías de especialización y de escala (en los países avanzados). Estos últimos se especializan en la provisión de componentes y servicios

\footnotetext{
${ }^{23}$ La llamada "patria contratista" es un concepto que describe las características de reproducción de la burguesía local, asociadas a los contratos de obra pública (Basualdo, 2010).
} 

Año XXIV, N 38/Junio 2018, ISSN 2468-9912. DNDA: 5355295 en línea

Mariano Treacy

Discusiones sobre la autonomía en la recepción de los aportes dependentistas al campo de las Relaciones Internacionales y contribuciones recientes desde el enfoque de Cadenas Globales de Valor

Págs. 45-67

sofisticados y de alta tecnología mientras que los países emergentes se vinculan principalmente con actividades intensivas en el uso de mano de obra, con un menor valor agregado unitario. Por lo tanto, lejos de ser neutral, la estrategia global de segmentación productiva adoptada por las firmas transnacionales generará ganadores y perdedores en términos de la distribución de los costos y beneficios.

La dependencia, en este contexto, adquiere nuevas características, ya que se asume dimensiones tecnológicas, industriales y financieras. Si bien se ha modificado la apariencia, el contenido de la dependencia se mantiene. A través de la conformación de Cadenas Globales de Valor (CGV) ${ }^{24}$ (Gereffi, 2001) se configuran relaciones económicas entre espacios nacionales ordenadas jerárquicamente y dominadas por el comercio intrafirma o intrasectorial. Este ordenamiento favorece el abastecimiento al sector productivo local de los insumos y tecnología necesaria sin desarrollar necesariamente segmentos de agregación de valor (Kosacoff y López, 2008).

Los patrones de desarrollo local o regional en las CGV deben ser entendidos como partes constitutivas de un proceso global de desarrollo desigual en los que la reproducción de los países centrales se basa en una captura de valor desconectada del desarrollo productivo que generan en los países donde se emplazan (Carballa, Durand y Knauss, 2016). Esta segmentación productiva genera en los hechos una división intemacional del trabajo en la cual firmas independientes se incorporan a redes de producción en las que las funciones vinculadas a la organización del proceso de trabajo y la apropiación del beneficio son distribuidas de forma desigual. Las firmas líderes de las CGV acceden a organizar el proceso de trabajo (tecnología, estándares laborales, etc.) hacia el interior de la red y ostentan la capacidad de internalizar o transferir precios a lo largo de la cadena, lo que les permite capturar parte de los beneficios generados en entidades formalmente autónomas.

Los beneficios asociados a la participación en una CGV no se vinculan necesariamente con las mejoras en la eficiencia derivadas de la inversión productiva sino que tienen que ver con la capacidad de apropiarse de los distintos tipos de renta en función del poder de

\footnotetext{
${ }^{24}$ Según Kaplinsky y Morris (2001), la cadena de valor describe el rango completo de actividades que se requieren para hacer un producto, desde su concepción atravesando las distintas fases de producción (que involucran una transformación física y el uso de servicios) hasta su entrega a los consumidores finales y su tratamiento luego de su uso.
} 
Revista Aportes para la Integración Latinoamericana Año XXIV, Nº 38/Junio 2018, ISSN 2468-9912. DNDA: 5355295 en línea

Mariano Treacy

Discusiones sobre la autonomía en la recepción de los aportes dependentistas al campo de las Relaciones Internacionales y contribuciones recientes desde el enfoque de Cadenas Globales de Valor

Págs. 45-67

mercado, las barreras a la entrada, las estructuras asimétricas, la protección de estándares o de derechos de propiedad intelectual, etc. ${ }^{25}$. Estos segmentos son de difícil acceso a empresas que no cuentan con ventajas competitivas o un vínculo estratégico con las firmas líderes. Los segmentos competitivos, donde es relativamente más "sencillo" insertarse, son precisamente aquellos donde se produce y se transfiere el excedente (Carballa, Durand y Knauss, 2016).

La especialización productiva de los países emergentes entonces radicará básicamente en estas opciones: 1) Importar manufacturas intermedias (con alto contenido tecnológico) que luego exportan como bienes finales a los países centrales ${ }^{26}$; o 2) Exportar bienes o servicios vinculados a ventajas comparativas ${ }^{27}$. América Latina se inserta débilmente en actividades de Investigación y Desarrollo $(I+D)$ o servicios empresariales basados en tecnología. Se concentra principalmente en la explotación de materias primas y el acceso al mercado local (Kosacoff y López, 2008). La inserción internacional está basada fundamentalmente en ventajas comparativas estáticas vinculadas a los recursos naturales en Sudamérica y a los bajos costos laborales y a los recursos naturales en América Central y el Caribe. Si se analizan las productividades relativas y el ritmo del progreso tecnológico de los productos exportados y las tasas de crecimiento de los mercados de destino de las exportaciones se identifica también un retroceso en comparación con el núcleo dinámico del sudeste asiático ${ }^{28}$. Las exportaciones de bienes de alto contenido

\footnotetext{
${ }^{25}$ Siguiendo la caracterización de Gereffi (2001), se distinguirán distintos tipos de renta en función de la cadena productiva en la que opere. Las empresas que operan en las Cadenas Productivas dirigidas al Productor (CPP) generalmente podrán capturar rentas asociadas al desarrollo tecnológico, rentas organizativas y rentas vinculadas a la producción a escala. Las empresas que operan en las Cadenas Productivas Dirigidas al Consumidor (CPC) en general podrán capturar rentas vinculadas a las relaciones que establecen con otras empresas y las redes que construyen, rentas de política comercial y rentas de la marca, más vinculadas al diseño del producto y al alcance que tengan para llegar al consumidor final.

${ }^{26}$ La importación de bienes intermedios en los países periféricos es sumamente relevante para entender la dinámica del comercio internacional y de la organización mundial de la producción. Como muestra de ello, el $73 \%$ de las importaciones de China en 2007 fueron bienes intermedios, porcentaje que ascendió al $81 \%$ en Malasia, al $80 \%$ en Tailandia, al $79 \%$ en Filipinas y al $66 \%$ en México (Minian, 2009).

${ }^{27}$ Recordemos que las ventajas comparativas representan la teoría tradicional del comercio internacional y compara los costos de producción en condiciones de autarquía para demostrar que el comercio internacional es mutuamente beneficioso incluso en aquellos casos en los que un país posea menores costos absolutos en la producción de ambos bienes. Las nuevas teorías que se basan en esta idea añaden diferencias tecnológicas y diferencias relativas al precio de los factores productivos (Krugman, 2001).

${ }^{28}$ El caso del desarrollo de los tigres asiáticos fue largamente estudiado. Evans (1998) señala que se dio la particularidad de una "autonomía enraizada" donde una burocracia estatal capacitada se articuló con la comunidad empresarial en la construcción de un proyecto nacional de desarrollo, evitando apropiaciones de renta mediante el disciplinamiento del sector privado. En este sentido, la capacidad de un Estado de sostener una trayectoria de desarrollo exitosa depende de una serie de conexiones externas y vinculaciones internas
} 

Año XXIV, N 38/Junio 2018, ISSN 2468-9912. DNDA: 5355295 en línea

Mariano Treacy

Discusiones sobre la autonomía en la recepción de los aportes dependentistas al campo de las Relaciones Internacionales y contribuciones recientes desde el enfoque de Cadenas Globales de Valor

Págs. 45-67

tecnológico tienen una proporción muy baja de integración local y la dinámica de innovación se sitúa fuera de los países.

El abaratamiento de los costos derivado de la segmentación productiva y la producción de manufacturas en países emergentes incide decisivamente en la competitividad de la industria de los países centrales, desde donde se promueve este proceso de relocalización. Mientras que las exportaciones estandarizadas de bienes completos o insumos intermedios está sujeta a una fuerte competencia que presiona sus precios a la baja, la producción de bienes no estandarizados de alto nivel tecnológico que requieren también buenas remuneraciones y una alta capacitación de la mano de obra continúan siendo nichos donde la competencia opera de una forma distinta ya que no se da vía precios y existen barreras a la entrada, lo que permite la apropiación de rentabilidades extraordinarias. La inserción en las redes de segmentación de la producción global implica por ello una distribución desigual de los beneficios en favor de aquellos segmentos con altas barreras a la entrada, capaces de retener los retornos y en contra de aquellos segmentos, cuya competitividad depende de los costos salariales y la competencia vía precio, con bajo acceso a la tecnología y a los procesos de automatización.

La estrategia de industrialización, en este contexto, queda reducida a la "captura" del conocimiento internacional mediante la importación de bienes de capital y bienes intangibles y el fomento al ingreso de inversiones extranjeras. Esta estrategia es, a todas luces, una estrategia dependiente, ya que no genera los mecanismos para lograr un salto productivo ni una reubicación en los segmentos internacionales en los que se divide el trabajo. Para peor, no permite capturar el excedente que se genera, que se transfiere a quienes concentran el poder en las cadenas. El margen para emprender procesos de industrialización independiente con mayor grado de autonomía es sustancialmente menor hoy que hace treinta años, y absolutamente menor hoy que hace cincuenta años.

El sistema internacional sigue teniendo reglas y continúa estando estratificado, pero es claro que los Estados deben "compartir" (ya que realidad expresan) su capacidad de acción con otros actores no estatales, como las empresas transnacionales o los

para consolidar objetivos institucionales independientes, a la vez que se produce un enraizamiento con el sector privado para responder a las necesidades. 

Año XXIV, N 38/Junio 2018, ISSN 2468-9912. DNDA: 5355295 en línea

Mariano Treacy

Discusiones sobre la autonomía en la recepción de los aportes dependentistas al campo de las Relaciones Internacionales y contribuciones recientes desde el enfoque de Cadenas Globales de Valor

Págs. 45-67

organismos multilaterales estatales (el consejo de seguridad de la Organización de las Naciones Unidas, la Organización del Tratado del Atlántico Norte) o no estatales (como el Fondo Monetario Internacional, la Organización Mundial del Comercio, el Banco Mundial).

\section{CONCLUSIONES}

En este trabajo se realizó un balance crítico de la forma en la que se apropiaron los aportes de la Teoría de la Dependencia por parte del campo de las Relaciones Internacionales y se identificaron algunos elementos contemporáneos que podrían llegar a ser incorporados. La elección de la Teoría de la Dependencia se basa en que ha sido considerada por muchos analistas una de las principales escuelas que contribuyeron desde el sur global con aportes novedosos y relevantes a las discusiones sobre Relaciones Internacionales y Política Exterior.

En la configuración actual del capitalismo, Estado, Empresa Transnacional y Elites Locales se vinculan de modo tal que se han modificado las condiciones de la autonomía. La política exterior de los Estados se encuentra condicionada por los intereses y estrategias de las Elites Locales pero también permeada por las estrategias de segmentación internacional e integración productiva de las Empresas Transnacionales. Además, la relación entre Estados es jerárquica y la consolidación de Organismos Internacionales y Multilaterales ha provocado una cesión de soberanía que ha reducido aún más el margen de maniobra de los Estados periféricos o emergentes.

En este contexto, la apuesta por estrategias de política exterior autonómicas como salida al atraso y la dependencia, como fueran postuladas por autores como Puig y Jaguaribe, deben entenderse en el marco de las relaciones de poder y el modo en que funciona la producción y la circulación de bienes y servicios en el mundo actual.

La Escuela Latinoamericana de la Autonomía puso el eje en el rol de las élites y los condicionantes a la política exterior y planteó una estrategia centrada en garantizar la independencia del Estado mediante la generación de instrumentos para defender la soberanía nacional. El Estructuralismo cepalino, por su parte, basó su estrategia en la necesidad de promover una industrialización que mediante la sustitución de importaciones de bienes estratégicos y tecnología permitiera sostener un sendero de desarrollo 

Año XXIV, N 38/Junio 2018, ISSN 2468-9912. DNDA: 5355295 en línea

Mariano Treacy

Discusiones sobre la autonomía en la recepción de los aportes dependentistas al campo de las Relaciones Internacionales y contribuciones recientes desde el enfoque de Cadenas Globales de Valor

Págs. 45-67

ampliando de esta forma la autonomía nacional con respecto a la dependencia industrial, tecnológica y financiera del extranjero.

Compartiendo parte de estos diagnósticos, la Teoría de la Dependencia entendió, sin embargo, la autonomía y las estrategias de desarrollo en el marco de las relaciones estructurales de poder que se establecen entre las élites locales, el capital extranjero y los Estados. Estas relaciones de poder se cristalizan en una división internacional del trabajo que en ningún caso resulta neutral sino que tiene ganadores y perdedores derivados de componentes históricos, políticos y económicos

En su vinculación con el Capital Extranjero, América Latina no logró consolidar una trayectoria autónoma de desarrollo ni un bloque en el poder con interés autonomista debido a la forma en la que se integró en la división internacional del trabajo. La extranjerización de la estructura productiva no condujo a un escalamiento productivo ni a la generación de mayor valor agregado local sino que se ha concentrado en la explotación de ventajas comparativas. Esta situación ha reproducido las relaciones de dependencia ya que los países de la región experimentan, desde entonces, recurrentes crisis externas vinculadas a la necesidad de financiar la importación de insumos, bienes de capital y tecnologías del exterior y a la incapacidad de contar con fuentes genuinas de financiamiento para hacerlo. Las élites locales, lejos de ser un eje de resistencia a la reproducción de este modelo de acumulación, han colaborado y se han convertido en aliadas estratégicas en este proceso.

Las posibilidades de desarrollar una estrategia de autonomía continúan estando condicionadas por la configuración del orden internacional y por el control científicotecnológico y financiero y la hegemonía cultural de un puñado de actores. El régimen internacional y la división global del trabajo, así como también la configuración de alianzas internas entre las élites, condicionan las alternativas que poseen los países periféricos para "insertarse en el mundo" y lograr posiciones mayores de autonomía participando en la gobernanza global.

Cualquier tipo de estrategia de política exterior y de proyecto de desarrollo autonómico debe considerar el modo en que la configuración de la dependencia en la actualidad restringe el margen para elaborar, implementar y sostener políticas autonómicas. Un 

Año XXIV, Nº 38/Junio 2018, ISSN 2468-9912. DNDA: 5355295 en línea

Mariano Treacy

Discusiones sobre la autonomía en la recepción de los aportes dependentistas al campo de las Relaciones Internacionales y contribuciones recientes desde el enfoque de Cadenas Globales de Valor

Págs. 45-67

cambio hacia una política exterior autonómica no puede pensarse si no es de la mano de un cambio en las condiciones estructurales que reproducen la dependencia.

\section{BIBLIOGRAFÍA}

Arceo, E. (2005). El impacto de la globalización en la periferia y las nuevas y viejas formas de la dependencia en América Latina. Cuadernos del CENDES, 22(60), 27-63.

Bambirra, V. (1999). El capitalismo dependiente latinoamericano. Ciudad de México, México: Siglo XX.

Barneix, P., Forcinito, K., \& Treacy, M. (2014). Aportes de la sociología de la cultura para pensar la relación existente entre producción científica de pensamiento económico y dominación social. En Wainer, V. La enseñanza de la economía en el marco de la crisis del pensamiento económico., Los Polvorines, Argentina: Ediciones UNGS.

Basualdo, E. (2010). Estudios de historia económica argentina: desde mediados del siglo XX a la actualidad. Buenos Aires, Argentina: Siglo XXI - FLACSO.

Borón, A. (2008). Teoría(s) de la dependencia. Realidad Económica (238), 20-43.

Bourdieu, P. (2002). Campo de poder, campo intelectual. Itinerario de un concepto. Buenos Aires, Argentina: Montressor.

Briceño Ruiz, J., \& Simonoff, A. (2017). La Escuela de la Autonomía, América Latina y la teoría de las relaciones internacionales. Estudios internacionales (Santiago), 49(186), 3989.

Carballa Smichowski, B., Durand, C., \& Knauss, S. (2016). "Uneven development patterns in global value chains. An empirical inquiry based on a conceptualization of GVCs as a specific form of the division of labor" (No. 2016-06). Centre d'Economie de l'Université de Paris Nord. Disponible en: https://ideas.repec.org/p/upn/wpaper/2016-06.html

Cardoso, F. H., \& Faletto, E. (1969). Desarrollo y dependencia en América Latina. Ciudad de México, México: Siglo XXI. 

Año XXIV, Nº 38/Junio 2018, ISSN 2468-9912. DNDA: 5355295 en línea

Mariano Treacy

Discusiones sobre la autonomía en la recepción de los aportes dependentistas al campo de las Relaciones Internacionales y contribuciones recientes desde el enfoque de Cadenas Globales de Valor

Págs. 45-67

Constantino, A., \& Cantamutto, F. J. (2014). Patrón de reproducción del capital y clases sociales en la Argentina contemporánea. Sociológica (México), 29(81), 39-86.

Cueva, A. (1974). Problemas y perspectivas de la teoría de la dependencia. En: Entre la ira y la esperanza y otros ensayos de crítica latinoamericana. Fundamentos conceptuales (2008). Bogotá, Colombia: Siglo del Hombre - CLACSODos Santos, T. (1971). La estructura de la dependencia. En Sweezy, P.; Wolff, R.; Dos Santos, T. y Magdoff, H. Economía política del imperialismo. Buenos Aires, Argentina: Periferia.

Dos Santos, T. (2011). Imperialismo y dependencia. Caracas, Venezuela: Fundación Biblioteca Ayacucho.

Escudé, C. (2012). El realismo periférico (RP) y su relevancia teórica ante el ascenso de China. Desarrollo Económico,51 (204), 529-542.

Evans, P. (1998). Alternativas al Estado desarrollista: Lecciones de la crisis de Asia Oriental. Nueva Sociedad, 155, 142-156.

Gereffi, G. (2001). Las cadenas productivas como marco analítico para la globalización. Problemas del Desarrollo. Revista Latinoamericana de Economía, 32(125), 9-37.

Kaplinsky, R., \& Morris, M. (2001). A handbook for value chain research (Vol. 113). Idrc, Ottawa.

Kosacoff, B., \& López, A. (2008). América Latina y las cadenas globales de valor: debilidades y potencialidades. Revista de Globalización, Competitividad y Gobernabilidad, 2(1).

Krugman, P., Obstfeld, M., Garuz, J. T., Diaz, E. S., \& Taña, J. O. (2001). Economía internacional. Pearson education.

Legler, T., Santa Cruz, A., \& González, L. Z. (2016). Introducción a las relaciones Internacionales. Ciudad de México, México: Oxford University Press.

Marini, R. M. (1973). Dialéctica de la dependencia. Ciudad de México, México: Era. 

Año XXIV, N 38/Junio 2018, ISSN 2468-9912. DNDA: 5355295 en línea

Mariano Treacy

Discusiones sobre la autonomía en la recepción de los aportes dependentistas al campo de las Relaciones Internacionales y contribuciones recientes desde el enfoque de Cadenas Globales de Valor

Págs. 45-67

Marini, R. M. (2008). Proceso y tendencias de la globalización capitalista y otros textos (Antología). Prometeo Libros Editorial, Buenos Aires.

Míguez, M. C. (2017). La autonomía heterodoxa y la clasificación de las políticas exteriores en la Argentina. Revista de relaciones internacionales, estrategia y seguridad, 12(2), 207.

Míguez, M. C., \& Deciancio, M. (2016). La internacionalización de la teoría de las Relaciones Intemacionales en la Argentina: los híbridos teóricos y su clasificación. Papeles de trabajo: La revista electrónica del IDAES, 10(18), 169-192.

Minian, I. (2009). Nuevamente sobre la segmentación internacional de la producción. Economía UNAM, 6(17), 46-68.

Palma, G. (1987). Dependencia y desarrollo: una visión crítica. Seers, D. La teoría de la dependencia. Una revaluación crítica. Fondo de Cultura económica, México, 21-89.

Robinson, W. I. (2007). Una teoría sobre el capitalismo global: Producción, clases y Estado en un mundo transnacional. Ediciones desde abajo, Bogotá.

Slipak, A. (2016). Ruy Mauro Marini, un imprescindible para el debate latinoamericano. Cuestiones de Sociología, 14, e007.

Schteingart, D., \& Coatz, D. (2015). ¿Qué modelo de desarrollo para la Argentina?. Boletín informativo TECHINT, 349(2.2), 1, 49-88.

Tarcus, H. (2007). Marx en la Argentina. Sus primeros lectores obreros, intelectuales y científicos. Siglo XXI, Buenos Aires.

Tickner, A. (2009). Latin America: Still Policy Dependent after all these Years?. Tickner, A. International Relations Scholarship around the World. Routledge, London.

Tickner, A. (2011). Relaciones de conocimiento centro-periferia: hegemonía, contribuciones locales e hibridación. Godoy, H. Gonzalez, R, Orozco, G., Drekonja-Kornat, G. Construyendo lo global: aporte al debate de relaciones internacionales. Editorial Universidad Del Norte, Barranquilla. 
Tickner, A. B. (2013). Core, periphery and (neo) imperialist International Relations. European Journal of International Relations, 19(3), 627-646.

Treacy, M. (2015). Dependencia, restricción externa y transferencia de excedente en la Argentina (1970-2013). Cuadernos De Economía Crítica, 1(2), 113-139.

Treacy, M. (2016). "Nuevas formas de dependencia y de integración regional en tiempos de mundialización neoliberal”. Densidades (Buenos Aires) 20,69-97.

Tussie, D. (2015). Relaciones Internacionales y Economía Política Internacional: notas para el debate. Relaciones Internacionales (La Plata) 28, 155-175.

Treacy MARIANO: Investigador y Docente de la Universidad Nacional de General Sarmiento (UNGS). Licenciado en Economía (UBA), Magister en Economía Política (FLACSO) y Candidato a Doctor en Ciencias Sociales (UBA). Integrante de la Sociedad de Economía Crítica (SEC). Correo electrónico: mtreacy@ungs.edu.ar 